\title{
In vivo demonstration of injectable microstimulators based on charge-balanced rectification of epidermically applied currents
}

\author{
Antoni Ivorra $^{1,2}$, Laura Becerra-Fajardo ${ }^{2}$, Quim Castellví ${ }^{2}$ \\ ${ }^{1}$ Serra Húnter Fellow \\ ${ }^{2}$ Department of Information and Communication Technologies, Universitat Pompeu \\ Fabra, Barcelona, Spain \\ E-mail: antoni.ivorra@gmail.com
}

\begin{abstract}
.
Objective. It is possible to develop implantable microstimulators whose actuation principle is based on rectification of high frequency (HF) current bursts supplied through skin electrodes. This has been demonstrated previously by means of devices consisting of a single diode. However, previous single diode devices caused DC currents which made them impractical for clinical applications. Here flexible thread-like stimulation implants which perform charge balance are demonstrated in vivo. Approach. The implants weight $40.5 \mathrm{mg}$ and they consist of a $3 \mathrm{~cm}$ long tubular silicone body with a diameter of $1 \mathrm{~mm}$, two electrodes at opposite ends and, within the central section of the body, an electronic circuit made up of a diode, two capacitors and a resistor. In the present study, each implant was percutaneously introduced through a $14 \mathrm{G}$ catheter into either the gastrocnemius muscle or the cranial tibial muscle of a rabbit hind limb. Then stimulation was performed by delivering $\mathrm{HF}$ bursts (amplitude $<60 \mathrm{~V}$, frequency $1 \mathrm{MHz}$, burst repetition frequency from $10 \mathrm{~Hz}$ to $200 \mathrm{~Hz}$, duration $=200 \mu \mathrm{s}$ ) through a pair of textile electrodes strapped around the hind limb and either isometric plantarflexion or dorsiflexion forces were recorded. Stimulation was also assayed 1, 2 and 4 weeks after implantation. Main results. The implants produced bursts of rectified current whose mean value was of a few $\mathrm{mA}$ and were capable of causing local neuromuscular stimulation. The implants were well tolerated during the 4 weeks. Significance. Existing power supply methods, and in particular inductive links, comprise stiff and bulky parts. This hinders the development of minimally invasive implantable devices for neuroprostheses based on electrical stimulation. The proposed methodology is intended for relieving such bottleneck. In terms of mass, thinness and flexibility, the demonstrated implants appear to be unprecedented among the intramuscular stimulation implants ever assayed in vertebrates.
\end{abstract}




\section{Introduction}

Implantable stimulation systems consisting of central units wired to stimulation electrodes are not adequate for applications in which a large number of targets must be individually stimulated over large and mobile body parts, thus hindering solutions for patients suffering from paralysis due to spinal cord injury or other neurological disorders [1]. In this kind of scenarios, besides the complexity of the surgical implantation process, further drawbacks of centralized stimulation systems are manifested: the long and bulky leads running from the active electronics to the stimulation electrodes are prone to mechanical failure, may cause mechanical damage to tissues and may promote infections [2,3].

An alternative to centralized stimulation systems can consist of network of addressable single-channel wireless microstimulators which are deployed with simple implantation procedures, such as injection, and which are wirelessly activated in coordinated patterns by an external automated controller. This kind of solution was proposed and tried in the past [3-5]. However, previously developed microstimulators are stiff and considerably large (diameters $>2 \mathrm{~mm}$ ) which makes them unsuitable for some applications because of their invasiveness [6].

Further miniaturization has been prevented until now because of the use of inductive coupling and batteries as energy sources. Inductive coupling largely depends on the area of the coil contained within the implant. Even when multiple turns and a ferrite core are used for implementation, the coil still needs to be considerably wide; it is difficult to theoretically conceive devices based on inductive coupling with diameters significantly below $1 \mathrm{~mm}$ [7]. In a recent publication it has been demonstrated a variant of near-field inductive coupling which consists in confining electromagnetic energy transport through electromagnetic waves in tissue [8]. Nevertheless, even with this novel approach - which should make possible to power deep-seated miniature implants - the diameter of the coil will have to be above $1 \mathrm{~mm}$. In particular, the publication demonstrates an implant that weights $70 \mathrm{mg}$ and which contains a $2 \mathrm{~mm}$ diameter coil.

In [9] we proposed and demonstrated a heterodox method for performing electrical stimulation: implanted devices rectify bursts of innocuous high frequency (HF) currents supplied to the tissue of interest by remote electrodes so that low frequency (LF) excitatory currents are generated locally through the implants. The externally delivered auxiliary currents, which are ohmically conducted through the 
tissues, and which could be delivered by means of textile electrodes on a garment, are incapable of causing stimulation of excitatory tissues because of their high frequency $(>100 \mathrm{kHz})$ and they do not cause significant Joule heating because they are delivered as very short bursts (duty cycle $<5 \%$ ). On the other hand, the resultant currents through the implants, despite being much lower than the auxiliary currents, are capable of performing stimulation of local excitable tissues because of their low frequency contents (typically from $1 \mathrm{~Hz}$ to $100 \mathrm{~Hz}$ ). In comparison to inductive coupling - or to electrochemical batteries - this method offers an unprecedented potential for miniaturization. Note that only two electrodes are required both for picking-up the high frequency (HF) currents and for performing stimulation. In addition, all necessary electronic components can be integrated in a single integrated circuit or in a tiny hybrid microcircuit.

The proposed method requires a minimum voltage difference across the two implant electrodes for effective operation and this implies that, in most applications, it will be necessary that both electrodes are quite separated (from some millimeters to a very few centimeters). In [10] we showed that, in the hypothetical case of implants for neuromuscular electrical stimulation of the forearm muscles, the distance between the two electrodes has to be about $3 \mathrm{~cm}$ in order to be able to use auxiliary currents whose magnitude is low enough to meet safety standards. Consequently, we propose elongated thread-like implant bodies in which most of their length consists of flexible and stretchable materials whose density and mechanical properties match those of neighboring living tissues for minimizing tissue damage, tethering and implant dislocations [11].

For demonstrating the method in [9] the electronics consisted of a single commercial Schottky diode. Such a simple rectifier - which may be extremely small does not allow the above referred neuroprosthetic system consisting of a network of addressable wireless microstimulators. For achieving that, digitally addressable rectifiers will be required; their development constitutes one of our research interests [12]. However, simple non-addressable rectifiers could in principle be applicable to some clinical scenarios where the size of the stimulator matters and only a single target must be stimulated. Unfortunately, implants whose electronics consists of just a diode are not adequate for clinical applications because they cause DC currents through them. Passage of DC current through an electrode is known to cause electrochemical reactions that damage both the electrodes and the tissues [13]. For that reason, charge-balanced 
waveforms, resulting in a null DC component through the electrodes, are typically employed in electrical stimulation systems.

Here we in vivo demonstrate simple thread-like stimulation implants which perform charge balance for preventing electrochemical damage.

\section{Methods}

\subsection{The implants}

The design and the implementation of the implants used in this study are described in [14], where it is also in vitro demonstrated that the implants do perform charge balance thereby preventing electrochemical reactions. Here, for the convenience of the reader, the main aspects regarding the operation, design and the implementation of the implants are succinctly presented.

The implants weight $40.5 \mathrm{mg}$ and they consist of a $3 \mathrm{~cm}$ long tubular silicone body with a diameter of $1 \mathrm{~mm}$, two electrodes at opposite ends and, within the central section of the silicone body, an electronic circuit capable of performing charge-balanced rectification.

The circuit architecture is represented in Figure 1. The implant circuit is drawn in black whereas a simplified lumped element model corresponding to the rest of the system (i.e. external generator, external skin electrodes and tissue impedances) is drawn in gray. The nodes $a$ and $b$ represent the electrodes of the implant whose impedances are neglected for the sake of simplicity.

When $C_{B}$ is discharged, if a sinusoidal HF current burst of frequency $f=1 / T$ is forced to flow through the tissue in which the implant is located, the voltage across the implant electrodes will correspond to the half-wave rectification of the HF voltage that would appear across the electrodes if the diode was not present (amplitude $=A_{a b}^{H F}$ ). Assuming an ideal diode, the mean voltage of that rectified signal will be $-A_{a b}^{H F} / \pi$. Since cell membranes behave as low pass filters with cutoff frequencies in the order of a few $\mathrm{kHz}$ [15], the implant will be seen by the excitatory cells - in terms of membrane depolarization or hyperpolarization - as a LF voltage generator with magnitude $-A_{a b}^{H F} / \pi$ because the high frequency contents of the implant voltage (that is, components at $f$ and multiples of $f$ ) will be filtered out, provided that $f>>1 \mathrm{kHz}$. If the HF current is delivered in short bursts of duration $W$ and repetition frequency $F$, rather 
than continuously, the voltage from the implant will be seen by the cells roughly as square pulses of duration $W$ and frequency $F$, provided that $W>>T$ and $F<<f$.

During the HF bursts, rectified current will flow from $a$ to $b$ through the diode thus progressively charging $\mathrm{C}_{\mathrm{B}}$. This capacitor will prevent any flow of DC current through the implant electrodes. As $\mathrm{C}_{\mathrm{B}}$ becomes charged, higher voltage differences between $a$ and $b$ will be required in order to obtain conduction through the diode and, as a consequence, the mean voltage across the implant electrodes will decrease. Therefore, the generated LF pulse will not be square but it will show a decaying shape. Once the HF burst finishes, the capacitor will slowly discharge through the implant resistor $R_{D}$ and the tissues until the next burst.

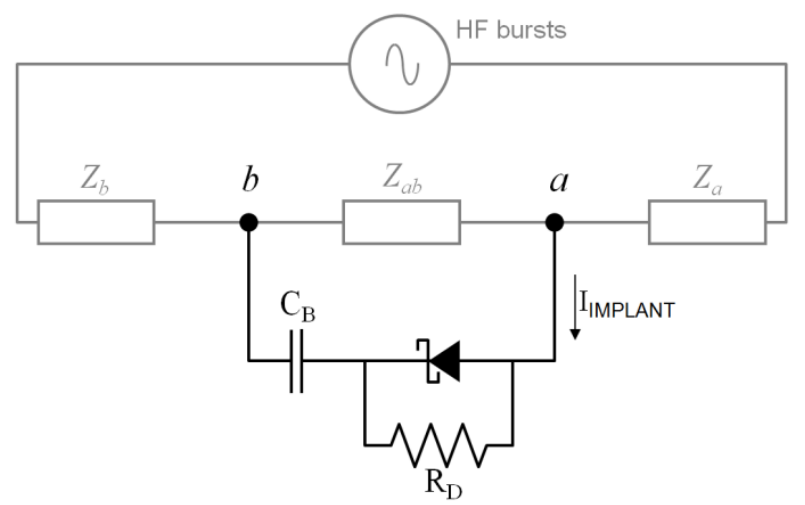

Figure 1. Implant circuit (in black) for performing charge-balanced rectification. The additional elements (in gray) are a simplified model of the rest of the system components: the external generator, the external skin electrodes and the tissue impedances.

A capacitor with a large enough capacitance value must be employed so that rectified current flows for a significant amount of time during the HF burst. In other words, a large capacitance is required in order to allow flow of enough charge for achieving stimulation. The capacitance value is mainly limited due to size constraints: not many submillimetric capacitors exist with capacitances above $1 \mu \mathrm{F}$ and the capability to withstand some volts or tens of volts as it is required in this application. The implants used in this study contain two $1.2 \mu \mathrm{F}$ capacitors in series, resulting in a combined capacitance of $0.6 \mu \mathrm{F}$ (Figure 2). This not only was done for doubling the voltage tolerance $(6.3 \mathrm{~V}+6.3 \mathrm{~V})$ but also for facilitating implant fabrication by achieving a symmetrical structure. 


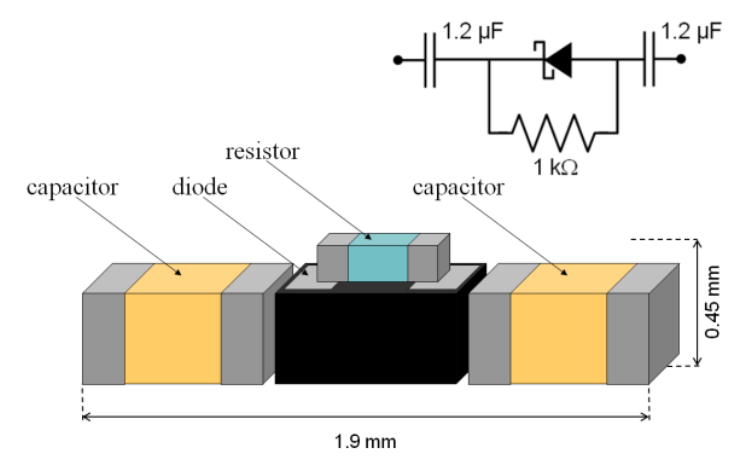

Figure 2. Placement of electronic components in the circuit of the implant. Two capacitors are employed in order to double the voltage tolerance $(6.3 \mathrm{~V}+6.3 \mathrm{~V})$ and for geometrical symmetry.

The capacitor must fully - or almost fully - discharge in between HF bursts. Once the value of $\mathrm{C}_{\mathrm{B}}$ is set, the discharge time constant depends on the implant resistor $\mathrm{R}_{\mathrm{D}}$. On the one hand, the resistance of this resistor cannot be too small; otherwise the amplitude of the HF voltage to be rectified $\left(A_{a b}^{H F}\right)$ will be too low because of shortcircuiting. On the other hand, the resistance cannot be extremely large; otherwise the capacitor will not fully discharge in between bursts. For an implant with a length of about $3 \mathrm{~cm}$, it can be determined that, taking into account the dielectric properties of tissues and the desired timing characteristics of the HF bursts $(W=200 \mu \mathrm{s}$ and $F<$ $200 \mathrm{~Hz}$ ), a $R_{D}$ in the order of $1 \mathrm{k} \Omega$ is adequate. (This will be demonstrated in a numerical study under preparation).

The implants used in this study (Figure 3) were implemented as follows: two SMD capacitors with a measured capacitance of $1.2 \mu \mathrm{F}(02016 \mathrm{D} 225 \mathrm{MAT} 2 \mathrm{~A}$ by AVX and acquired from Mouser Electronics; supposedly $2.2 \mu \mathrm{F}$ capacitors), a SMD Schottky diode (RB521ZS-30 by RHOM Semiconductor) and a SMD $1 \mathrm{k} \Omega$ resistor (ERJXGNJ102Y by Panasonic) were positioned as indicated in Figure 2 and soldered thus forming the proposed circuit. Two $\sim 2 \mathrm{~cm}$ long Pt-Ir springs were soldered to the circuit; one to each terminal. (These springs were made by coiling 41 AWG Pt-Ir wire (76700 by A-M Systems) around a 30-gauge needle.) The assembly was gently introduced in a $3 \mathrm{~cm}$ long biomedical silicone tube with an outside diameter of $0.94 \mathrm{~mm}$ (806400 by AM Systems). The tube was filled with a biocompatible silicone (MED-6015 by NuSil Technology, Carpinteria, CA, USA) which has a low viscosity when uncured. After curing, the Pt-Ir wire exiting the tube was bent inwards on the tube and another Pt-Ir spring (made with a 19-gauge needle) was tightly positioned over it to form an electrode of approximately 3 to $4 \mathrm{~mm}$ (one at each opposite end). Finally, a few drops of silicone were added and cured for securing the electrodes. 


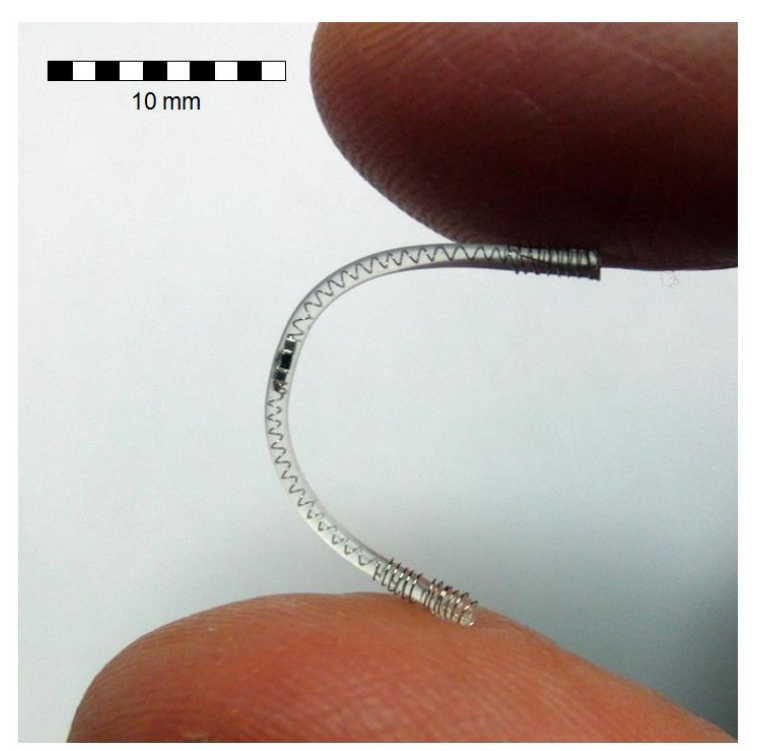

Figure 3. Picture of the implant used in this study. The electronic components and the two connections to the electrodes are contained within a silicone body. Both the connections and the electrodes consist of coiled Pt-Ir wires.

After implementation, these prototypes were electrically tested and the $a$ electrode (as defined in Figure 1) was identified and marked with ink. This electrode is from where negative LF currents are delivered to tissue during the HF burst and, therefore, is the electrode most likely to cause stimulation since excitatory tissues are more sensitive to negative (cathodic) currents from a nearby electrode than to positive currents [16].

Finally, the prototypes were rinsed and immersed for 5 minutes in ethanol $70 \%$ for sterilization and kept in sterile containers before their implantation days later.

\subsection{In vivo study}

\subsubsection{Animal handling}

The in vivo study was approved by the Ethical Committee for Animal Research (CEEAPRBB) (application number: JMC-14-1606). Three New Zealand White (NZW) male rabbits weighting from $3.5 \mathrm{~kg}$ to $4 \mathrm{~kg}$. were employed. Before and after the acute procedures each animal was stabled in a $0.7 \times 0.7 \mathrm{~m}^{2}$ cage $(\mathrm{R}$-Suite $\mathrm{X}$-Type Cage by TECNIPLAST S.p.A., Buguggiate, Italy) with ad libitum feeding and water.

Initial sedation and anesthesia for acute procedures (implantation and stimulation tests) consisted of Dexmedetomidine (15 mg/kg), Butorfanol (150 $\mu \mathrm{g} / \mathrm{kg})$ and Ketamine $(0.4 \mathrm{mg} / \mathrm{kg})$ intramuscularly administered. Before implantation, and if 
considered necessary before the subsequent stimulation tests, the hind limb was carefully shaved (from the head of the femur to the mid tarsus) to ensure good electrical dry contact between the external textile electrodes (later referred) and the skin. Then, the animal was transferred to an anesthetic circuit using endotracheal intubation and anesthesia was maintained with Sevofluorane 3-4\%. During the entire procedure Ringer's lactate was administered intravenously, capnography and pulse oximetry were constantly monitored and a heating pad was employed.

At the end of the study, the animals were euthanized with an overdose of Dolethal $(4 \mathrm{~mL} \mathrm{IV})$. The muscles where the devices were implanted were surgically extracted and preserved in formaldehyde 10\%. Later, blocks containing sections of the implants and the tissues surrounding them were cut and sent to an external laboratory (Histovet, Sant Quirze del Vallès, Barcelona, Spain) for preparation of histological sections (hematoxylin and eosin stain) and histological analysis.

\subsubsection{Implantation procedure}

The implantation procedure is illustrated in Figure 4. After selecting the muscle in which to deploy the implant (either the gastrocnemius muscle or the cranial tibial muscle), the muscle was identified by palpation and anatomical cues and a catheter with an external diameter of $2.1 \mathrm{~mm}$ (14 G, BD Angiocath model 382268 by Becton, Dickinson and Company, Franklin Lakes, NJ, USA) was longitudinally introduced from a location close to the hock up to the proximal end of the muscle, resulting in an insertion depth from about 5 to $7 \mathrm{~cm}$. In the last implantation (within the gastrocnemius) the procedure was slightly modified: the insertion point was about $2 \mathrm{~cm}$ below the muscle belly.

Then, an $\mathrm{Ag} / \mathrm{AgCl}$ gel electrode (model 2228 by 3M, St. Paul, MN, USA) was positioned on the thigh of the animal and conventional stimulation was attempted between the gel electrode and the catheter introducer needle (Figure $4 \mathrm{~b}$ ). That is, the tip of the catheter - in which the stainless steel introducer needle is exposed - was employed as an exploration electrode for locating the motor point where the implant would be later released. Stimulation signals consisted of 2 to $5 \mathrm{~V}$ bipolar square pulses of $200 \mu \mathrm{s}+200 \mu \mathrm{s}$ (cathodic first, no interphase dwell time) at $50 \mathrm{~Hz}$ and were delivered by means of a custom made generator. If the induced movement was deemed not strong enough or did not match the aimed movement pattern (dorsiflexion or plantarflexion) then the catheter was repositioned. Typically, catheter repositioning 
consisted in just pushing it in or pulling it out a few millimeters. Only in two occasions the catheter was completely pulled out and reinserted.

Once an adequate motor point was located, the insertion needle was pulled out and an implant prototype ( $a$ electrode first) was introduced through the catheter and pushed - with the help of a sterilized plastic cable with distance markings ("pusher") up to the tip of the catheter. Then, cautiously holding the plastic cable, the catheter was pulled out so that the implant was released inside the muscle. Finally, the plastic cable was pulled out and the insertion point was treated. Either no bleeding occurred or was minimal.

A total of 4 implantations were performed: tibial in rabbit 1 (left), gastrocnemius (right) and tibial (left) in rabbit 2 and gastrocnemius (left) in rabbit 3.

In one case, instead of deploying an implant, a wired bipolar probe was deployed so that external measurements could be performed on a circuit equal to that contained within the implants. The bipolar probe consisted in a $1.17 \mathrm{~mm}$ diameter coaxial cable (Filotex ET087059 by Nexans S.A., Paris, France). The core conductor (silver plated copper covered steel wire with a diameter of $0.17 \mathrm{~mm}$ ) was exposed for $3 \mathrm{~mm}$ at its distal tip and a $3 \mathrm{~mm}$ long piece of stainless steel tube with an outer diameter of $1.3 \mathrm{~mm}$ was placed in contact with the shield conductor at a distance of $3 \mathrm{~cm}$ from the tip. This formed a probe that consisted of two cylindrical electrodes at a distance of $3 \mathrm{~cm}$ on a flexible shaft.

After a series of stimulation assays (see next section), X-ray images of the hind limb of the animal were acquired.
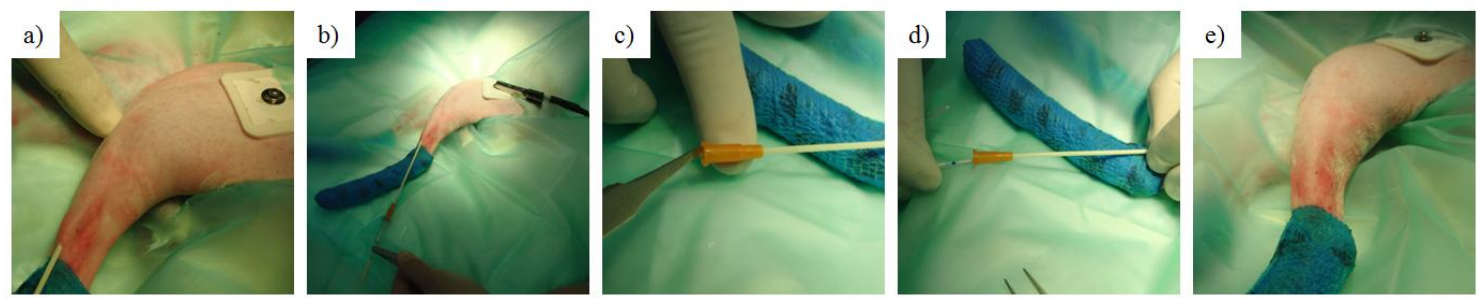

Figure 4. Implantation procedure. a) The muscle of interest was identified and a catheter with an external diameter of $2.1 \mathrm{~mm}$ was longitudinally introduced into it. b) For locating the motor point, conventional stimulation was attempted between the tip of the catheter needle and an external electrode. c) After pulling out the needle of the catheter, the implant was pushed through the catheter. e) Holding the pusher, the catheter was pulled out so that the implant was released inside the muscle. e) Finally, the pusher was pulled out and the insertion point was treated.

\subsubsection{Stimulation assays}

Stimulation assays were performed immediately after implantation and 1, 2 and 4 weeks after implantation. Here the procedure is described. 
A pair of band electrodes (about $2 \mathrm{~cm}$ wide) made of conductive fabric (MedTex P-180 by Statex, Bremen, Germany) were strapped around the hind limb where the implant to be activated was present (Figure 5). Those two bands were positioned as parallel as possible but, due to anatomical constraints, they were angled at $30^{\circ}$ or more. The minimum separation distance ranged from about $3 \mathrm{~cm}$ to $4 \mathrm{~cm}$ and the maximum separation distance ranged from about $5 \mathrm{~cm}$ to $7 \mathrm{~cm}$.

With the animal lying sideways, the hock was tied to an acrylic board by means of a padded cable tie and the hind foot was tied to a load cell (STC-10kgAL-S by Vishay Precision Group, Malvern, PA, USA) mounted on the board for measuring isometric dorsiflexion and plantarflexion forces. (Up to a point this force measurement setup was inspired by the torque measurement setup used in [17]).

In the first stimulation session, the contour of the textile electrodes was marked with a permanent marker on the rabbit skin and later, after the session was completed and still under anesthesia, the contour was tattooed with India ink so that the same external electrode location could be used in the following sessions.

Assays consisted in delivering a number of high frequency bursts and recording induced forces. The HF bursts $(f=1 \mathrm{MHz}, T=200 \mu \mathrm{s})$ were delivered to the textile electrodes by means of a function generator (AFG 3022B by Tektronix Inc., Beaverton, OR, USA) followed by a high voltage amplifier (WMA-300 by Falco Systems BV, Amsterdam, The Netherlands). Amplitudes (A) varied from $20 \mathrm{~V}$ to $60 \mathrm{~V}$ and burst repetition frequencies $(F)$ from $10 \mathrm{~Hz}$ to $200 \mathrm{~Hz}$. In all cases, delivery of the bursts was monitored with an oscilloscope (TPS2014 by Tektronix).

A custom developed virtual instrument (LabVIEW based and interfacing with an ACQ board NI USB-6211, both by National Instruments Corp., Austin, TX, USA) triggered the delivery of the HF bursts and recorded the measurements (sampling frequency $=50 \mathrm{kHz}$ ) from the load cell (through a custom developed signal conditioning electronics which included a first order low pass filter with a cutoff frequency of $500 \mathrm{~Hz}$ ). 
a)
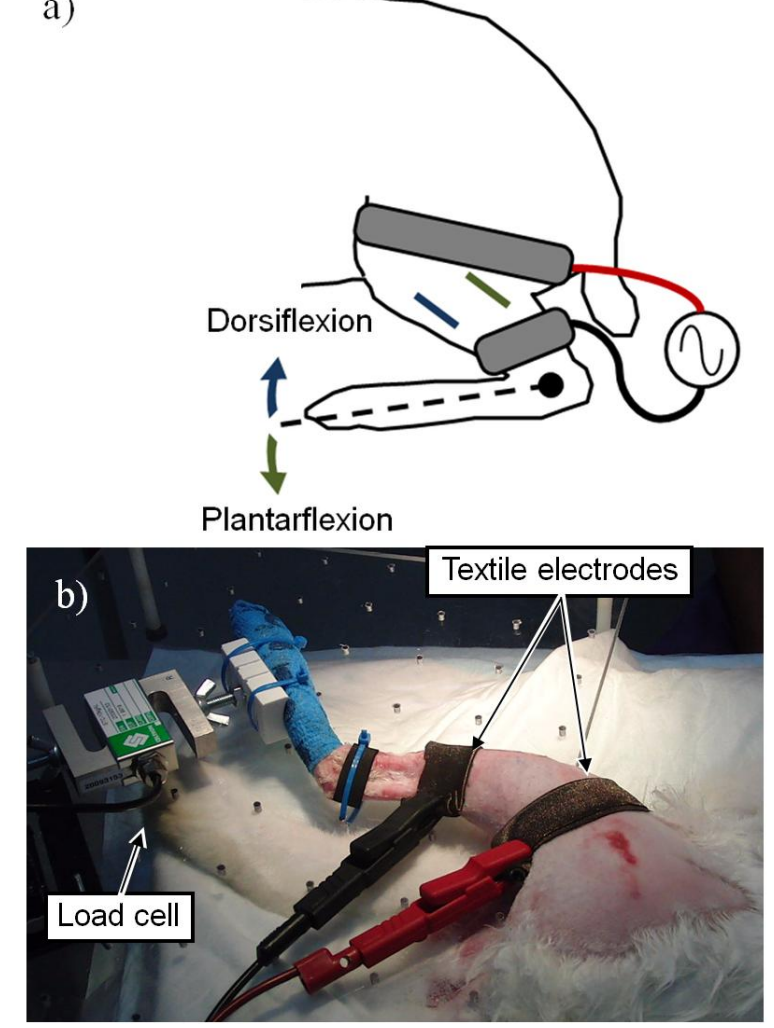

Figure 5. Setup employed for performing the isometric stimulation assays reported here. a) Schematic representation of the two possible locations of the implant and the corresponding movements. b) Picture from an actual experiment. The hind limb was tied to a rigid board and to a load cell for recording forces. Two textile electrodes were strapped around the hind limb for delivering the auxiliary HF current bursts.

Stimulation assays were also attempted in hind limbs where no implants were located in order to verify that the HF bursts were not able to induce any sort of movement or reaction.

\section{Results}

\subsubsection{The implants cause local neuromuscular stimulation}

Per se, the HF voltage bursts delivered across the external electrodes were incapable of producing any noticeable stimulation when no implants were deployed. On the other hand, when an implant was deployed inside the hind limb, either dorsiflexion or plantarflexion forces - depending on the location of the implant - were clearly observable. For illustration, the supplementary video contains sample recordings in which it is possible to clearly observe muscle contractions and movements.

Figure 6 shows a set of isometric force recordings for an implant deployed in the tibial muscle (rabbit 1). In these recordings, performed in the same stimulation session 
(2 weeks after implantation), the repetition rate $(F)$ of the $\mathrm{HF}$ bursts $(f=1 \mathrm{MHz}$, $T=200 \mu \mathrm{s}, A=50 \mathrm{~V}$ ) varied from $20 \mathrm{~Hz}$ to $200 \mathrm{~Hz}$. The recordings at $20 \mathrm{~Hz}$ and $50 \mathrm{~Hz}$ illustrate the summation effect of individual twitch contractions. At $100 \mathrm{~Hz}$ the muscle contraction is smooth. By increasing the frequency of the bursts (to $200 \mathrm{~Hz}$ ) a slight increase in force magnitude is obtained (peak force increases from $2.6 \mathrm{~N}$ at $100 \mathrm{~Hz}$ to $2.9 \mathrm{~N}$ at $200 \mathrm{~Hz})$.
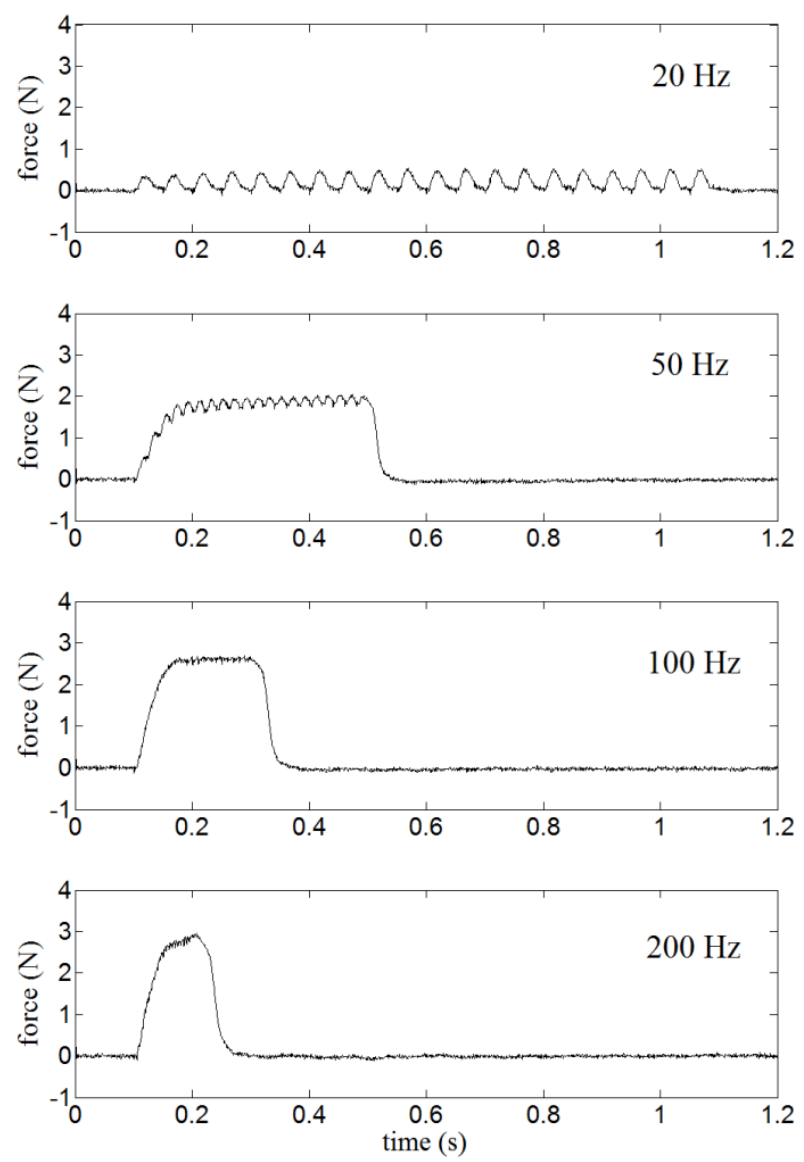

Figure 6. Isometric force recordings obtained by acting on an implant deployed in the tibial muscle (rabbit 1). The repetition frequency $(F)$ of the $\mathrm{HF}$ bursts $(f=1 \mathrm{MHz}, A=50 \mathrm{~V}, W=200 \mu \mathrm{s})$ delivered across the external electrodes was increased from $20 \mathrm{~Hz}$ to $200 \mathrm{~Hz}$. The number of HF bursts is fixed at 20.

\subsubsection{The high frequency bursts induce current impulses through the implants}

In one case, instead of deploying an implant, a wired bipolar probe was deployed so that external measurements could be performed on a circuit equal to that contained within the implants. The probe consisted of two cylindrical electrodes at a distance of $3 \mathrm{~cm}$ on a flexible shaft. Figure 7 shows oscilloscope recordings by means of that bipolar probe (deployed in gastrocnemius muscle).

Sinusoidal HF bursts ( $f=1 \mathrm{MHz}, A=50 \mathrm{~V}, T=200 \mu \mathrm{s}, F=100 \mathrm{~Hz}$ ) were delivered through the external textile electrodes (blue line in Figure 7b). When the 
circuit was connected, at the beginning of the HF burst, a half wave rectified signal with an amplitude of about $10 \mathrm{~V}$ appeared across the probe electrodes (black line in Figure $7 b)$.

As mentioned, stimulation is achieved thanks to the fact that the implants, by performing rectification, cause low frequency currents through them. To reveal those low frequency currents, a low pass filter for currents (first order, cutoff frequency = $7.2 \mathrm{kHz}$ ) was inserted in between the external circuit corresponding to the implant circuit and the probe (gray box in Figure 7c). The behavior of the recorded current (Figure 7d) coincides with the operation described in section 2.1: during the HF burst, low frequency current flows through the implant and the magnitude of this low frequency current diminishes as $C_{B}\left(C_{B 1}\right.$ in series with $\left.C_{B 2}\right)$ charges. In the recorded case the current goes from about $3 \mathrm{~mA}$ to $2.5 \mathrm{~mA}$. After the HF burst, the charge stored in $C_{B}$ is discharged through $R_{D}$ and the tissues thus causing a current of opposite sign that slowly diminishes.

a)

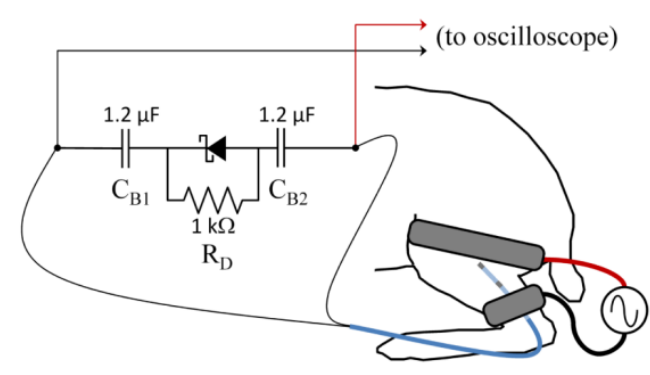

c)

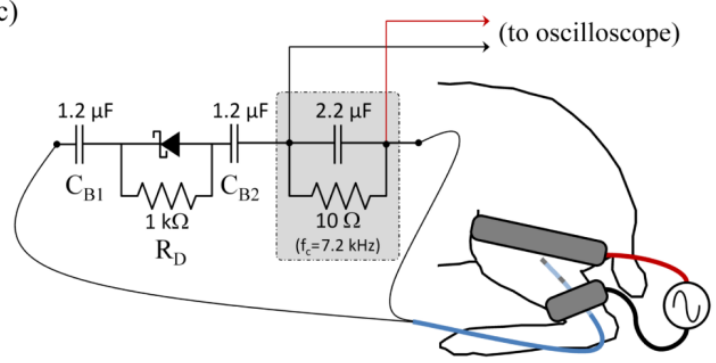

b)

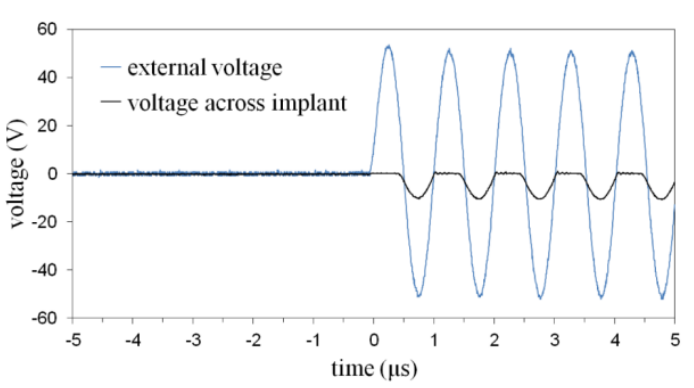

d)

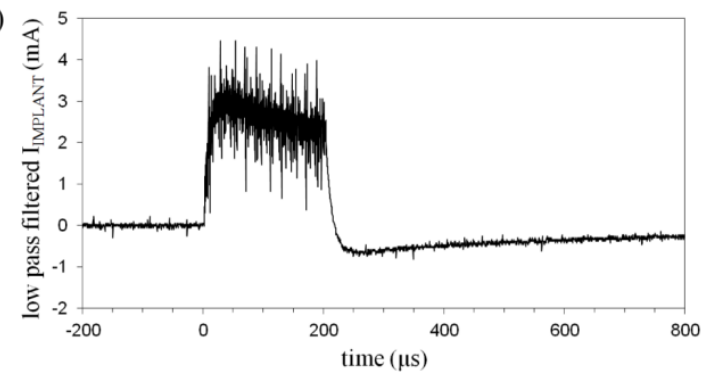

Figure 7. A wired bipolar probe implanted in a gastrocnemius muscle was used for performing measurements on a circuit equal to that contained within the implants. a) Setup for recording the voltage across the probe electrodes when the circuit is connected and stimulation occurs. b) Sinusoidal HF bursts ( $f=1 \mathrm{MHz}, A=50 \mathrm{~V}, W=200 \mu \mathrm{s}, F=$ $100 \mathrm{~Hz}$ ) were delivered across the external electrodes (blue line) and a half wave rectified voltage (black) appeared across the probe. c) Setup for recording the low frequency contents of the current through the implant. d) Low frequency current through the implant before, during and after a HF burst. 


\subsubsection{The implants are stable only if properly implanted}

Figure 8 shows force recordings at three consecutive stimulation sessions, across the span of two weeks, for the implant deployed in the left gastrocnemius muscle of rabbit 3. It can be observed that the generated forces are quite repetitive. However, not all the implants were as stable. While the four implanted prototypes were able to stimulate the desired muscles after implantation and all of them survived the first stimulation session (about 15 minutes of experiments), only two of them were still operative two weeks after implantation and at the end of the study (4 weeks) only one implant was still operative.
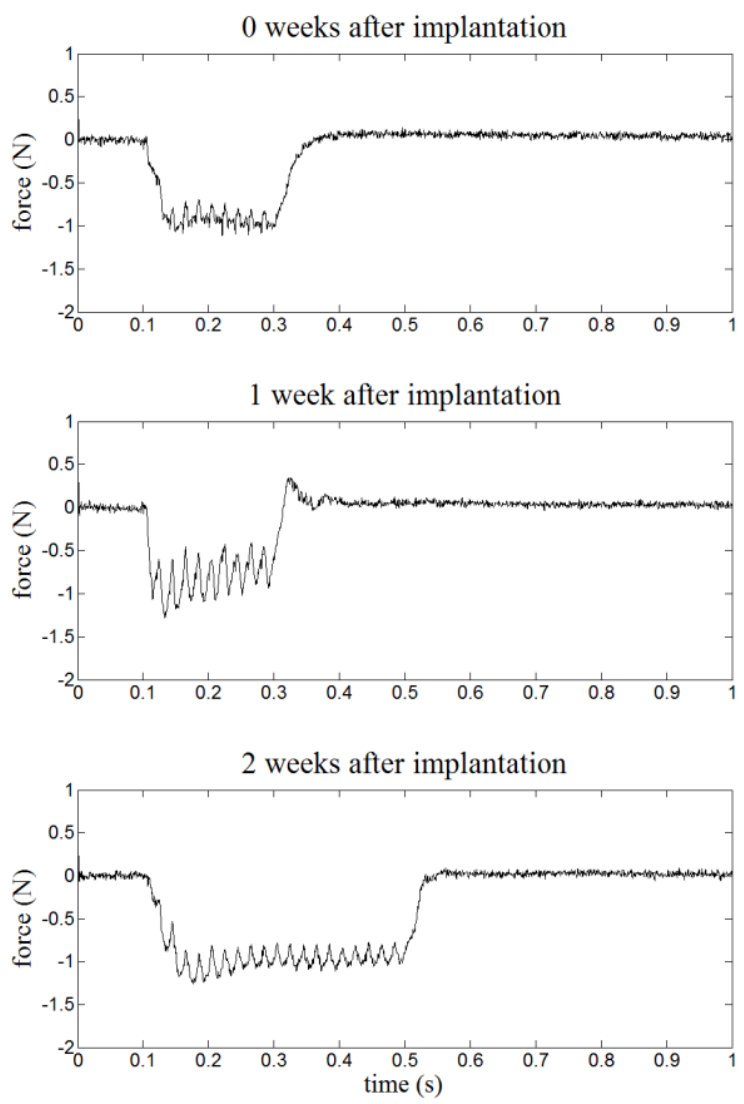

Figure 8. Evolution of isometric force recordings obtained by acting on the implant deployed in the left gastrocnemius muscle of rabbit 3 . The HF bursts $(f=1 \mathrm{MHz}, A=50 \mathrm{~V}, W=200 \mu \mathrm{s})$ were delivered at $50 \mathrm{~Hz}$ across the external electrodes. In the first two cases (immediately after implantation and 1 week after implantation) only 10 HF bursts were delivered whereas in the last case $20 \mathrm{HF}$ bursts.

X-ray images show that the implant deployed in the left gastrocnemius muscle of rabbit 3 was very stable (Figure 9). It did not move during the whole study. And it must be noted that during this period the animal was free to move and jump up and down a platform in the $0.7 \times 0.7 \mathrm{~m}^{2}$ cage. 
X-ray images of the other three implants showed that the implants were bent and significantly displaced from the site of deployment (Figure 10). This was observed just after the first stimulation session immediately after implantation. As argued later, in the discussion section, we hypothesized that this occurred because the implants were not wholly inserted into the target muscle but they crossed one or more muscle fascia and, as consequence, experienced large mechanical stresses. These observations motivated the above referred modification in the implantation process for the last implantation (in the left gastrocnemius muscle of rabbit 3): instead of introducing the catheter from a location close to the hook, the insertion point was about $2 \mathrm{~cm}$ below the muscle belly to ensure whole implantation within the gastrocnemius.

Three fatal damages of mechanical origin were noticed in the recovered implants: 1) the coiled wire electrode was pulled out, 2) one of the solder unions at the electronic circuit was broken and 3) the implant was cut off transversally.
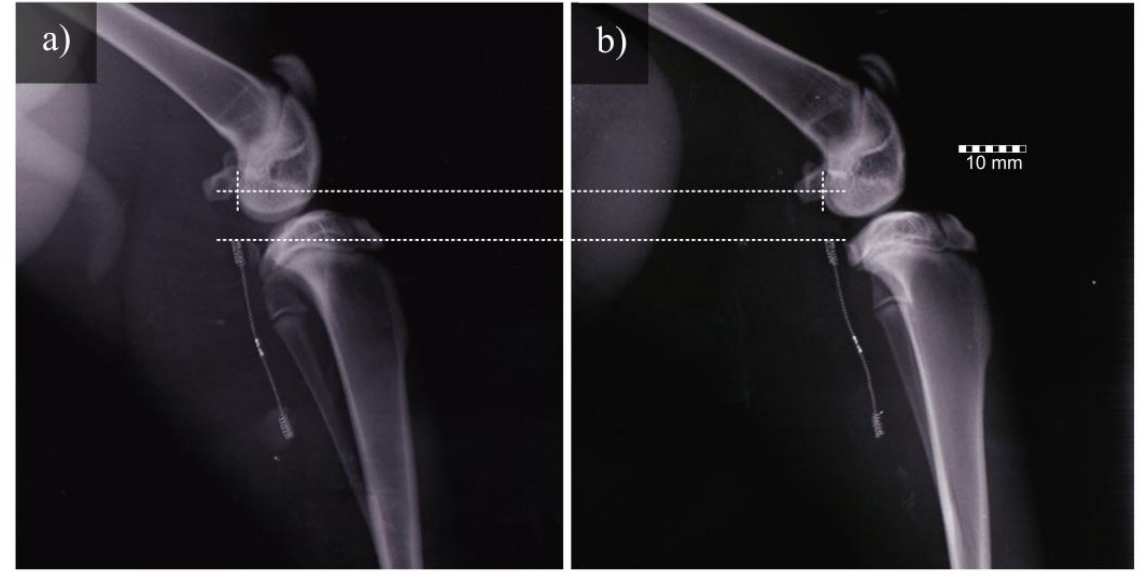

Figure 9. X-ray images of the implant in the left gastrocnemius muscle of rabbit 3. a) Just after implantation. b) Four weeks after implantation. The images were vertically aligned using for reference the apex of the round surface of the femoral condyles. No vertical displacement of the implant is appreciable. (The apparent slight lateral displacement most likely is due to differences in positioning while taking the images).
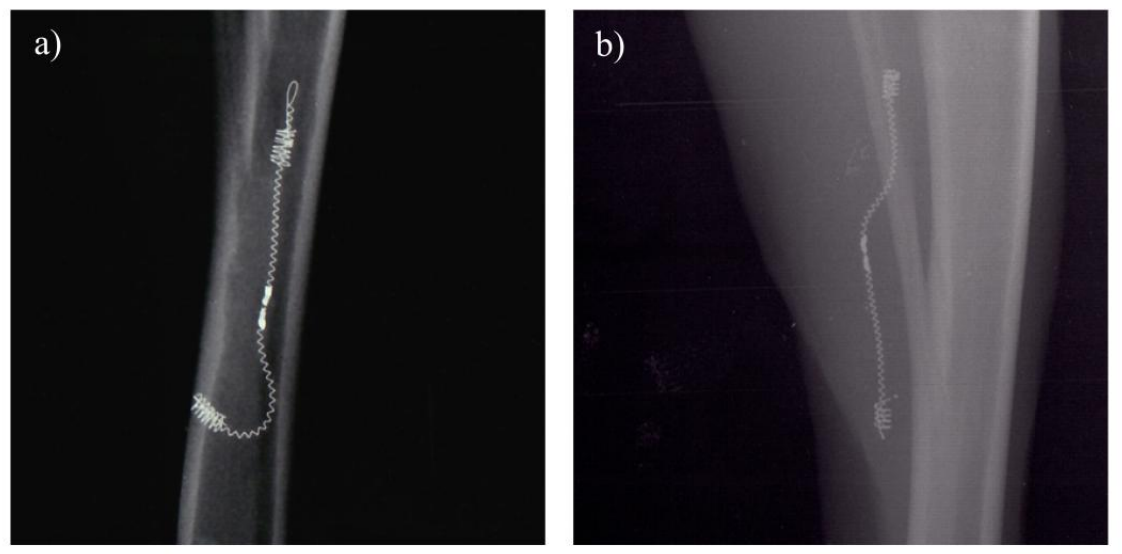

Figure 10. X-ray images of two of the implants that ended up failing. The implants are bent and significantly displaced from the site of deployment. Maybe, this occurred because the implants were not wholly inserted into the target muscle but they crossed one or more muscle fascia and, as consequence, experienced large mechanical stresses. 


\subsubsection{The implants are well tolerated}

No signs of discomfort were observed in the animals during the study. Figure 11 displays a transversal $H \& E$ section of the tissue surrounding the implant at the end of the study, that is, four weeks after implantation. In particular, this sample corresponds to the implant within the left gastrocnemius muscle of rabbit 3. An external and independent veterinary histopathologist (Histovet, Sant Quirze del Vallès, Barcelona, Spain) observed a local reaction in the vicinity of the implant characterized by the presence of mononuclear cells which is accompanied by moderate edema, fibrosis and re-epithelization. These are the expected foreign body response features at this stage [18]. No signs of mechanical damage were noticed when extracting the muscle samples for histology or in the histological preparations.

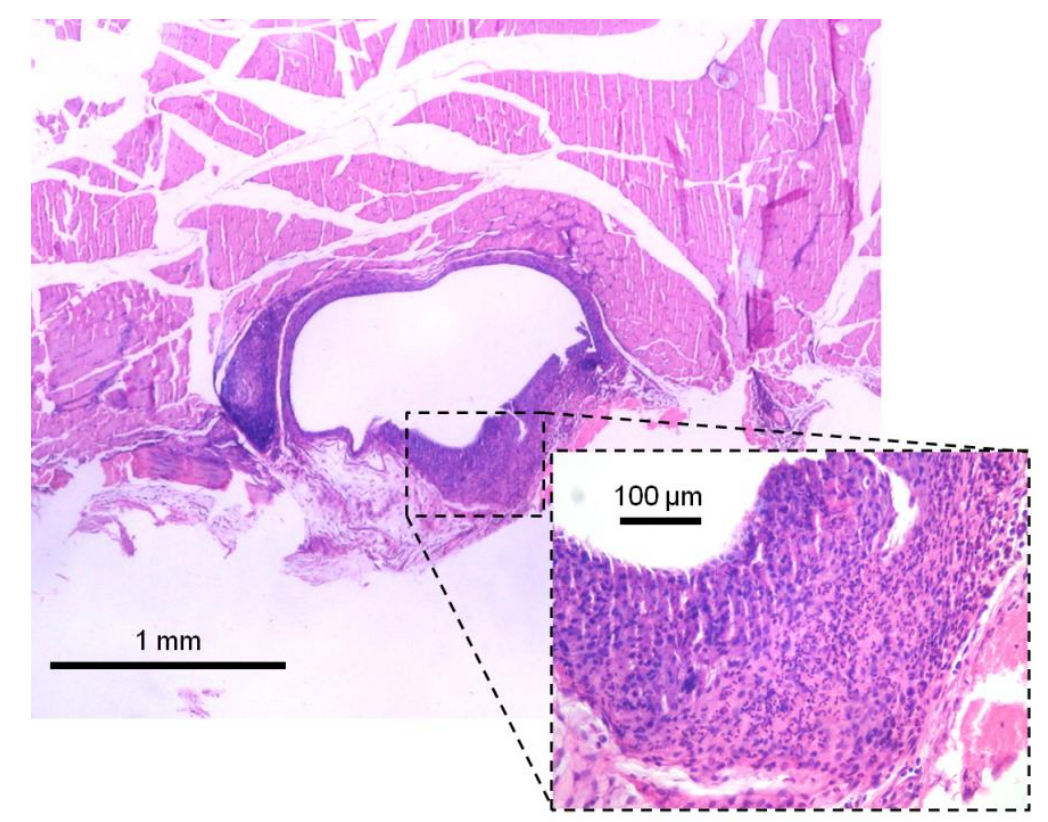

Figure 11. Histological section transversal to the implant in the left gastrocnemius muscle of rabbit 3 (4 weeks after implantation). (The implant segment came out before section preparation; that is why the void space is not circular, it partially collapsed during preparation).

\section{Discussion}

To the best of our knowledge, the demonstrated prototypes are unprecedented in terms of mass, thinness and flexibility among the intramuscular stimulation implants ever assayed in vertebrates. Submillimetric stimulators based on photodiodes have been proposed recently but these generate currents below $0.1 \mathrm{~mA}$ and, therefore, are only capable of stimulation if directly in contact with neurons [19]. That is, these photodiode based implants may be adequate for intraneural stimulation but not for intramuscular 
stimulation because, in this later case, the neurons to be excited may be some millimeters away from the electrode and much larger currents, in the order of a few mA, are required for stimulation.

In [9] we independently proposed and demonstrated stimulation based on rectification of ohmically conducted HF currents by testing implants consisting of just a diode. We later discovered that such stimulation method had already been proposed and demonstrated in vertebrates by at least two independent research teams in the 60s [20,21]. Nevertheless, those studies are barely cited and, in fact, no references to them are found after the 80s. More recently, also independently, the use of implanted RF diodes as rectifying stimulators powered by radiated electromagnetic fields - rather than through ohmic conductance as we propose - has also been demonstrated [22]. It must be noted, however, that such approach was already shown in studies by Schuder and colleagues in the $60 \mathrm{~s}[21]$.

Now we are proposing the development of more advanced circuits in order to implement electrical stimulation implants based on this method which will be adequate for clinical applications. In particular we are exploring the development of simple rectifiers capable of blocking DC currents (i.e. the contents of this paper) and of circuits with communication capabilities for implementing neuroprosthetic systems consisting of a network of addressable wireless microstimulators (see [12]). To the best of our knowledge, these efforts for exploiting the full potentially of the method are unprecedented.

This 4-week study indicates that the assayed implants, whose external parts are made of materials known to be biocompatible, are well tolerated by the rabbits. Nevertheless, it is not possible to extrapolate this conclusion to more prolonged implantations. Neither the implant design nor the study was intended to demonstrate long-term safety and operation of the implants.

Regarding long-term operation of the implants four issues at least should be analyzed: 1) evolution of the contact impedances between the implant electrodes and the tissues, 2) hermeticity and its impact on the electronics, 3) mechanical robustness of the implants and 4) anchorage of the implants to the site of deployment (immobility). Due to the limited duration of this study, no assumptions can be made from it regarding long-term evolution of the contact impedances; at 4 weeks the collagenous fibrotic capsule that develops around a material following implantation is just starting to grow [18]. Similarly, although in this study no failure was attributable to lack of hermeticity, 
little can be ensured concerning long-term survival of the electronic circuitry, which is embedded in a material known to be non-hermetic to water; although silicones have been successfully used for packaging simple circuitry in long-term implants [23], it seems plausible that the reduced dimensions of our implants may have a negative impact on the time to failure of the components. On the other hand, this study provides some hints regarding an aspect that may be crucial for long-term mechanical robustness of the implants and their immobility. It has been shown that a specific implant, which was entirely and longitudinally introduced into a single muscle, remained in place during the whole study. X-ray images of the other three implants, just after the first stimulation session, revealed that the implants were bent and significantly displaced from the site of deployment. In these three other cases it was not ensured that the implants would not be deployed crossing one or more muscle fascia. Actually, we are confident that that was the case. That is, these other implants were not wholly deployed into the target muscle. This is relevant because conventional electrode leads crossing muscle fascia have been reported to be subjected to great mechanical stresses and to be prone to failure [24,25]. The reason for this is that muscles experience longitudinal displacements with respect to neighbor muscles and, therefore, any object crossing an interface between them will be subjected to great mechanical stresses. Hence, we hypothesize that these relative displacements were the cause of the observed implant dislocations and of the fatal damages of mechanical origin. Aiming long-term operation, we conclude that it is advisable to deploy the implants longitudinally and entirely into the target muscle, avoiding crossings through muscle fascia. However, dislocation and mechanical damage could still appear even if the implant is deployed longitudinally and entirely into the target muscle. The muscle fibers surrounding the implant will shorten during contraction thus causing mechanical stresses which may propel the implant or damage it. Furthermore, if the implant is not completely aligned with the muscle fibers (i.e. it is partially transversal) additional shear stresses will be generated. Therefore, we plan improvements regarding anchorage mechanisms and robustness of the connections before attempting in vivo long-term assays. The results obtained with the implant that was entirely and longitudinally introduced into gastrocnemius muscle are encouraging because this device did not possess any intended anchorage mechanism besides the friction force by the silicone body and the electrodes. 


\section{Conclusions}

It is reconfirmed that local stimulation based on rectification of ohmically conducted bursts of high frequency current is feasible. In particular, the following specific conclusions can be drawn from this study:

Bursts $(W=200 \mu \mathrm{s}, F$ from $10 \mathrm{~Hz}$ to $200 \mathrm{~Hz}$ ) of high frequency sinusoidal voltage $(f=1 \mathrm{MHz}, A<60 \mathrm{~V})$ applied across two textile electrodes strapped around a depilated hind limb of a rabbit are incapable of producing any noticeable stimulation. On the other hand, after deploying a thread-like implant capable of electronic rectification into either the gastrocnemius muscle or the tibialis muscle of the hind limb, delivery of those bursts induces contraction of the corresponding muscle.

When a high frequency burst is delivered, the circuitry of the implants used in this study causes a burst of rectified current whose low frequency contents is equivalent to that of a quasi square pulse with an amplitude of a few $\mathrm{mA}$.

The thinness and elongated shape of the implant used in this study allows minimally invasive deployment into the muscles through a $14 \mathrm{G}$ catheter. In this 4 -week study, all the implants (4) were well tolerated by the rabbits.

\section{Acknowledgements}

The authors would like to express their gratitude to Jaume Banús (student at Universitat Pompeu Fabra, UPF) for his assistance in building the force measurement system, to Juan Martín Caballero (Director of the PRBB animal facility) for his assistance in writing the animal protocol and very specially to Sara Capdevila (Sub-director at the PRBB animal facility) and Jordi Grífols (Director at Zoològic Badalona Veterinària) for their excellent and crucial work regarding all animal procedures.

AI's research was supported by a Ramón y Cajal fellowship from the Spanish government and a Marie Curie grant (IRG 256376) from the European Commission. LB's research is supported by a scholarship from the UPF. QC's research is supported by a scholarship from the Spanish Ministry of Education.

\section{Competing financial interests}

The authors declare no competing financial interests.

\section{References}


[1] Mushahwar V K, Jacobs P L, Normann R A, Triolo R J and Kleitman N 2007 New functional electrical stimulation approaches to standing and walking $J$. Neural Eng. 4 S181-97

[2] Merrill D R, Davis R, Turk R and Burridge J H 2011 A personalized sensorcontrolled microstimulator system for arm rehabilitation poststroke. Part 1: System architecture Neuromodulation 14 72-9

[3] Kane M J, Breen P P, Quondamatteo F and OLaighin G 2011 BION microstimulators: a case study in the engineering of an electronic implantable medical device Med. Eng. Phys. 33 7-16

[4] Loeb G E, Zamin C J, Schulman J H and Troyk P R 1991 Injectable microstimulator for functional electrical stimulation Med. Biol. Eng. Comput. 29 NS13-9

[5] Ziaie B, Nardin M D, Coghlan A R and Najafi K 1997 A single-channel implantable microstimulator for functional neuromuscular stimulation IEEE Trans. Biomed. Eng. 44 909-20

[6] Li X, Serdijn W A, Zheng W, Tian Y and Zhang B 2015 The injectable neurostimulator: an emerging therapeutic device Trends Biotechnol. In Press

[7] Heetderks W J 1988 RF powering of millimeter- and submillimeter-sized neural prosthetic implants Biomed. Eng. IEEE Trans. 35 323-7

[8] Ho J S, Yeh A J, Neofytou E, Kim S, Tanabe Y, Patlolla B, Beygui R E and Poon A S Y 2014 Wireless power transfer to deep-tissue microimplants Proc. Natl. Acad. Sci.

[9] Ivorra A 2011 Remote electrical stimulation by means of implanted rectifiers PLoS One 6 e23456

[10] Ivorra A and Becerra-Fajardo L 2013 Wireless Microstimulators Based on Electronic Rectification of Epidermically Applied Currents: Safety and Portability Analysis Proceedings of the 18th IFESS Annual Conference ed T Keller and D B Popovic (Donostia-San Sebastián, Spain: ACADEMIC MIND, University of Begrade) pp 213-6

[11] Edell D J 2004 Insulating biomaterials Neuroprosthetics: Theory and Practice ed K W Horch and G S Dhillon (Singapore: World Scientific Publishing) pp 517-79

[12] Becerra-Fajardo L and Ivorra A 2015 In Vivo Demonstration of Addressable Microstimulators Powered by Rectification of Epidermically Applied Currents for Miniaturized Neuroprostheses PLoS One 10 e0131666

[13] Cogan S F 2008 Neural stimulation and recording electrodes. Annu. Rev. Biomed. Eng. 10 275-309 
[14] Ivorra A and Becerra-Fajardo L 2014 Flexible Thread-like Electrical Stimulation Implants Based on Rectification of Epidermically Applied Currents Which Perform Charge Balance Replace, Repair, Restore, Relieve - Bridging Clinical and Engineering Solutions in Neurorehabilitation vol 7 (Springer International Publishing) pp 447-55

[15] Santos-Sacchi J 1992 On the frequency limit and phase of outer hair cell motility: effects of the membrane filter. J. Neurosci. 12 1906-16

[16] Reilly J P 1998 Excitation models Applied Bioelectricity: From Electrical Stimulation to Electropathology ed J P Reilly (New York: Springer-Verlag) pp 105-47

[17] Riso R R, Mosallaie F K, Jensen W and Sinkjaer T 2000 Nerve cuff recordings of muscle afferent activity from tibial and peroneal nerves in rabbit during passive ankle motion IEEE Trans. Rehabil. Eng. 8 244-58

[18] Anderson J M, Rodriguez A and Chang D T 2008 Foreign body reaction to biomaterials Semin. Immunol. 20 86-100

[19] Abdo A, Sahin M, Freedman D S, Cevik E, Spuhler P S and Unlu M S 2011 Floating light-activated microelectrical stimulators tested in the rat spinal cord $J$. Neural Eng. 8056012

[20] Palti Y 1962 Stimulation of muscles and nerves by means of externally applied electrodes Bull. Res. Counc. Isr. E Exp. Med. 10 54-6

[21] Schuder J C and Stoeckle H 1964 The silicon diode as a receiver for electrical stimulation of body organs Trans. Am. Soc. Artif. Intern. Organs 10 366-70

[22] Towe B C, Larson P J and Gulick D W 2012 A microwave powered injectable neural stimulator Proceedings of the 2012 Annual International Conference of the IEEE Engineering in Medicine and Biology Society (EMBC), (San Diego, CA: IEEE) pp 5006-9

[23] Brindley G S, Polkey C E, Rushton D N and Cardozo L 1986 Sacral anterior root stimulators for bladder control in paraplegia: the first 50 cases. J. Neurol. Neurosurg. Psychiatry 49 1104-14

[24] Marsolais E B and Kobetic R 1986 Implantation techniques and experience with percutaneous intramuscular electrodes in the lower extremities. J. Rehabil. Res. Dev. $231-8$

[25] Handa Y, Hoshimiya N, Iguchi Y and Oda T 1989 Development of percutaneous intramuscular electrode for multichannel FES system. IEEE Trans. Biomed. Eng. $36705-10$ 